\title{
Investigation of the effect of nocodazole on the PI3K/Akt signaling pathway in COS7 cells
}

\author{
HUAIPING SHI ${ }^{1}$, WUJUN WU ${ }^{2}$ and JUN LUO ${ }^{1}$ \\ ${ }^{1}$ College of Animal Science and Technology, Northwest A\&F University, Yangling, Shaanxi 712100; \\ ${ }^{2}$ Department of Hepatobiliary Surgery, Shaanxi Provincial People's Hospital, Xi'an City, Shaanxi 710068, P.R. China
}

Received December 14, 2013; Accepted September 9, 2014

DOI: $10.3892 / \mathrm{mmr} .2014 .2632$

\begin{abstract}
A number of studies have demonstrated that nocodazole suppresses Akt phosphorylation; however, the underlying molecular mechanism remains unclear. In the current study, the mechanism of nocodazole-induced suppression of Akt phosphorylation was investigated. The cell cycle was analyzed using flow cytometry and EGF receptor dimerization was evaluated through a cross-linking assay. Immunoprecipitation experiments were performed to investigate the interaction between P85 and EGFR and cell migration was evaluated through a wound healing assay. COS7 cells were observed to be rounded following a 24-h treatment with nocodazole, and the results revealed that $\sim 45 \%$ of COS7 cells were arrested at the $\mathrm{G}_{2} / \mathrm{M}$ phase and that the cyclin $\mathrm{B} 1$ expression level was greatly increased. EGF-mediated Akt phosphorylation was markedly inhibited in nocodazole-treated cells. In addition, the levels of internalized EGF-EGFR complexes in nocodazole-treated cells were reduced, and EGF-EGFR dimerization was found to be affected by nocodazole. Akt phosphorylation in COS7 cells was demonstrated to be overridden by AG1478 and wortmannin. The results also showed that p85 did not bind to activated EGFR in nocodazole-treated cells, and that nocodazole and protein inhibitors reduced cell migration. In summary, these results indicate that nocodazole inhibits the PI3K/Akt pathway by interfering with the binding of p85 binding to activated EGFR and further affects the growth of cells.
\end{abstract}

\section{Introduction}

PI3-kinases (PI3Ks) have been linked to a diverse range of cellular functions, including the proliferation, differentiation and survival of cells. A number of these functions are associated with the ability of class $1 \mathrm{PI} 3 \mathrm{Ks}$ to activate protein

Correspondence to: Mr. Huaiping Shi, College of Animal Science and Technology, Northwest A\&F University, 22 Xinong Road, Yangling, Shaanxi 712100, P.R. China

E-mail: huaipingshi@nwsuaf.edu.cn

Key words: PI3K/Akt pathway, EGF, nocodazole, inhibitor kinase $\mathrm{B}$ ( $\mathrm{PKB}$ or $\mathrm{Akt})$. It has previously been reported that activated EGFR binds to and activates PI3K via its interaction with the p85 subunit (1-3). Once the class 1A family of PI3K is activated, PI3K phosphorylates its lipid substrate phosphatidylinositol 4,5-bisphosphate $\left[\operatorname{PtdIns}(4,5) P_{2}\right]$ and converts PtdIns $(4,5) P_{2}$ to phosphatidylinositol $(3,4,5)$-trisphosphate $\left[\operatorname{Ptd} \operatorname{Ins}(3,4,5) P_{3}\right]$, which is an important intracellular lipid second messenger. Subsequently, $\operatorname{PtdIns}(3,4,5) P_{3}$ recruits 3'-phosphoinositide-dependent protein kinase-1 (PDK1) and Akt to the plasma membrane. The colocalization of activated PDK1 and Akt allows for the phosphorylation of Akt at threonine (T)308 by PDK1 (4). The maximum activation of Akt requires the phosphorylation of T308 and a second site, serine (S)473, by PDK2 $(5,6)$. The activated PI3K/Akt pathway provides survival signals via several mechanisms, including the phosphorylation and inactivation of Bad and caspase-9, in addition to the stimulation of nuclear factor $-\kappa \mathrm{B}(\mathrm{NF}-\kappa \mathrm{B})$ activity (7-9). In the cell cycle, activation of the PI3K/Akt signaling pathway facilitates $\mathrm{G}_{1} / \mathrm{S}$ progression via the inactivation of glycogen synthase kinase-3 $\beta$ (GSK3- $\beta$ ) and promotes $\mathrm{G}_{2} / \mathrm{M}$ phase transition (10-12).

To date, a number of studies have indicated that the deregulation of the cell cycle via the over-activation of the PI3K/Akt signaling pathway may result in a variety of types of human cancers (13). Inhibition of the PI3K pathway prevents uncontrolled cellular proliferation and may control the growth of tumors. Consequently, the anti-apoptotic signals transduced by PI3K and downstream molecules have become a focus of recent studies into novel therapeutic strategies. LY-294002 and wortmannin are two widely used PI3K specific inhibitors. LY-294002 dephosphorylates PtdIns(3,4,5)P3, overrides PI3K signaling and significantly inhibits EGF-induced DNA synthesis (14). Wortmannin is able to bind to the p110 subunit of PI3K and specifically inactivate it (15). Furthermore, other PI3K inhibitors, such as AG1478 against EGFR phosphorylation, indirectly resist PI3K activity. In certain cases, antimitotic drugs, including nocodazole and paclitaxel, oppose the PI3K/Akt signaling pathway $(16,17)$. Previous studies determined that Akt activity was minimal in cells arrested by nocodazole treatment at the $\mathrm{G}_{2} / \mathrm{M}$ phase (11) and that nocodazole interfered with the PI3K/Akt signaling pathway (18). However, the mechanism by which nocodazole inhibits the PI3K/Akt signaling pathway remains undetermined. 
In the present study, the PI3K/Akt signaling pathway was investigated in nocodazole-treated cells in vitro. Additionally, the effects of nocodazole on cell growth were observed.

\section{Material and methods}

Cells lines and culture. COS7 fibroblast-like cells and 293T cells (Typical Culture Preservation Commission Cell Bank, Chinese Academy of Sciences, Shanghai, China) were grown at $37^{\circ} \mathrm{C}$ in Dulbecco's modified Eagle's medium (DMEM; Gibco-BRL, Carlsbad, CA, USA) containing 10\% fetal bovine serum (FBS; Hyclone, Logan, UT, USA), penicillin and streptomycin. The cells were maintained in a $5 \% \mathrm{CO}_{2}$ atmosphere.

Antibodies and chemicals. Monoclonal mouse anti-EGFR (Ab1) antibody was obtained from Oncogene Science (Cambridge, MA, USA), polyclonal rabbit anti-p85 (Z-8) was purchased from Upstate Biotechnology, Inc. (Lake Placid, NY, USA), monoclonal mouse anti-tubulin, polyclonal goat anti-P-EGFR (1086), polyclonal goat anti-Akt (N-19), polyclonal rabbit anti-P-Akt (Ser 473) and anti-cyclin B1 (H-433) were purchased from Santa Cruz Biotechnology (Dallas, TX, USA). Horseradish peroxidase-conjugated secondary antibodies (including polyclonal goat anti-rabbit Ig coupled to HRP, polyclonal rabbit anti-goat Ig coupled to HRP and polyclonal goat anti-mouse Ig coupled to HRP) and nitrocellulose membranes were purchased from Bio-Rad (Hercules, CA, USA) and EGF was obtained from Upstate Biotechnology. Nocodazole, AG1478 and TR-EGF were purchased from Calbiochem (Billerica, MA, USA), wortmannin was purchased from Alomone Labs (Jerusalem, Israel) and Texas Red (TR)-labeled EGF was purchased from Molecular Probes, Inc. (Camarillo, CA, USA). Unless otherwise specified, all chemicals were purchased from Sigma (St. Louis, MO, USA).

Morphology of nocodazole-treated cells. When the confluence of the COS7 cells reached $\sim 80 \%$ they were treated with or without $200 \mathrm{ng} / \mathrm{ml}$ nocodazole for $24 \mathrm{~h}$. Following nocodazole treatment, the cells were observed under an Olympus microscope BX51 equipped with a DP70 digital camera (Olympus, Tokyo, Japan).

Flow cytometric analysis. For flow cytometric analysis, each group of COS7 cells was collected by centrifugation at $500 \mathrm{xg}$ for $5 \mathrm{~min}$. Cells were fixed in precooled ethanol for $2 \mathrm{~h}$ on ice, suspended in RNase A solution $(0.2 \mathrm{mg} / \mathrm{ml}$ in $50 \mathrm{mM}$ Tris, $\mathrm{pH}$ 7.8) and incubated for $6 \mathrm{~h}$ at $37^{\circ} \mathrm{C}$. Samples were stained with $0.1 \mathrm{mg} / \mathrm{ml}$ propidium iodide (PI) for $30 \mathrm{~min}$. Finally, cells were sorted with a Cytomics FC500 flow cytometer (Beckman Coulter, Brea, CA, USA) to analyze the percentage of cells in each cell phase.

EGF-mediated Akt activity in response to nocodazole treatment. The effect of time on EGF treatment was investigated. Following overnight treatment with or without $200 \mathrm{ng} / \mathrm{ml}$ nocodazole, COS7 cells were stimulated by EGF at a final concentration of $50 \mathrm{ng} / \mathrm{ml}$ at the indicated times $(0,5,15,30$, 60 and $120 \mathrm{~min}$ ), followed by lysis of the cells and detection of Akt phosphorylation. Briefly, cells was lysed for $15 \mathrm{~min}$ at $4^{\circ} \mathrm{C}$ on a rocker using Mammalian Protein Extraction Kit (Cwbiotech, Beijing, China). The lysates were centifugated at $4^{\circ} \mathrm{C}$ for $15 \mathrm{~min}$ at $21000 \mathrm{x} \mathrm{g}$ and the supernatant was collected after the addition of SDS-loading buffer and heating at $95^{\circ} \mathrm{C}$ for $5 \mathrm{~min}$. The protein sample was separated by $10 \%$ SDS-PAGE to detect Akt phosphorylation using the phospho Akt antibody (Santa Cruz Biotechnology).

In addition, the effect of dose on EGF treatment was investigated. COS7 cells were treated with or without $200 \mathrm{ng} / \mathrm{ml}$ nocodazole overnight and stimulated for $15 \mathrm{~min}$ by EGF $(1,5,10$ or $50 \mathrm{ng} / \mathrm{ml})$. This was followed by lysis of the cells and detection of Akt phosphorylation.

Observation of internalized EGF-EGFR complexes (endosomes). COS7 cells grown on glass coverslips were treated for $24 \mathrm{~h}$ with or without $200 \mathrm{ng} / \mathrm{ml}$ nocodazole, followed by treatment with $50 \mathrm{ng} / \mathrm{ml}$ TR-EGF for $30 \mathrm{~min}$. The cells were washed twice with phosphate buffered saline (PBS), fixed with methanol and acetone $(1: 1)$ for $10 \mathrm{~min}$ at $-20^{\circ} \mathrm{C}$, and permeabilized with $0.2 \%$ Triton X-100 in PBS for 5 min prior to addition to a mounting solution. The cell endosomes were observed using a Nikon (EFD-3) fluorescence microscope (Nikon, Tokyo, Japan).

Cross-linking assay. 293T cells were cultured in 60-mm dishes to near confluency. Following transfection with EGFR-YFP, the cells were serum-starved for $24 \mathrm{~h}$. Subsequently, the cells were treated with EGF for $15 \mathrm{~min}$ at $37^{\circ} \mathrm{C}$ and collected in 0.2-0.5 ml PBS. Bis(sulfosuccinimidyl) suberate (BS3; Calbiochem, Dormstadt, Germany) was added to the cells to a final concentration of $1.0-2.5 \mathrm{mM}$ and the reaction was incubated on ice for $2 \mathrm{~h}$. A quenching solution (1 M Tris, $\mathrm{pH} 7.5,1: 100$ dilution) was added to a final concentration of $10 \mathrm{mM}$ and the mixture incubated for a further $15 \mathrm{~min}$ on ice. The cells were then lysed with a 1\% NP-40 solution (Abcam, Cambridge, UK) for $1 \mathrm{~h}$ on ice, and receptor dimerization was analyzed using PAGE and immunoblotting. The cell lysates were separated by electrophoresis using $7.5 \%$ polyacrylamide SDS-containing gels depending on the size of protein of interest (37.5:1 Acrylamide /bis, Tris-Cl; pH 8.8, TEMED, ammonium persulfate). Prestained protein markers were used for molecular weight standards. Proteins were electrophoretically transferred onto nitrocellulose membranes (BioRad, Hercules, CA). The transfer was done using a semi-dry blotting apparatus (Model SD transfer cell, BioRad) at $15 \mathrm{~V}$ per minigel for $45 \mathrm{~min}$ in transfer buffer. Blots were blocked with $3 \%$ skim milk in $0.05 \%$ Tween-PBS (blocking buffer) for $30 \mathrm{~min}$. Membranes were then probed with monoclonal mouse anti EGFR in blocking buffer overnight, and the primary antibodies were detected with a polyclonal goat anti-mouse Ig coupled to HRP followed by enhanced chemiluminescence, with SuperSignal ECL western Blotting Detection Reagents (Pierce, USA). The expression levels of EGFR protein were used to evaluate EGF receptor dimerization.

Observation of Akt phosphorylation in response to protein inhibitors. Following the addition of $0.5 \mu \mathrm{M}$ AG1478 or $100 \mathrm{nM}$ wortmannin to nocodazole-treated and -untreated cells for $30 \mathrm{~min}$, the cells were stimulated with $1 \mathrm{ng} / \mathrm{ml} \mathrm{EGF}$ for 15 min, lysed using Mammalian Protein Extraction Kit 
Table I. Flow cytometric analysis of the COS7 cell cycle following treatment with nocodazole.

\begin{tabular}{lccc}
\hline Group & $\mathrm{G}_{0} / \mathrm{G}_{1}(\%)$ & $\mathrm{S}(\%)$ & $\mathrm{G}_{2} / \mathrm{M}(\%)$ \\
\hline Control & $30.6 \pm 6.3$ & $61.2 \pm 5.6$ & $8.2 \pm 2.1$ \\
Nocodazole & $17.8 \pm 1.8^{\mathrm{a}}$ & $36.7 \pm 2.9^{\mathrm{a}}$ & $45.5 \pm 3.7^{\mathrm{a}}$ \\
\hline
\end{tabular}

${ }^{\mathrm{a}} \mathrm{P}<0.05$, compared with the control.

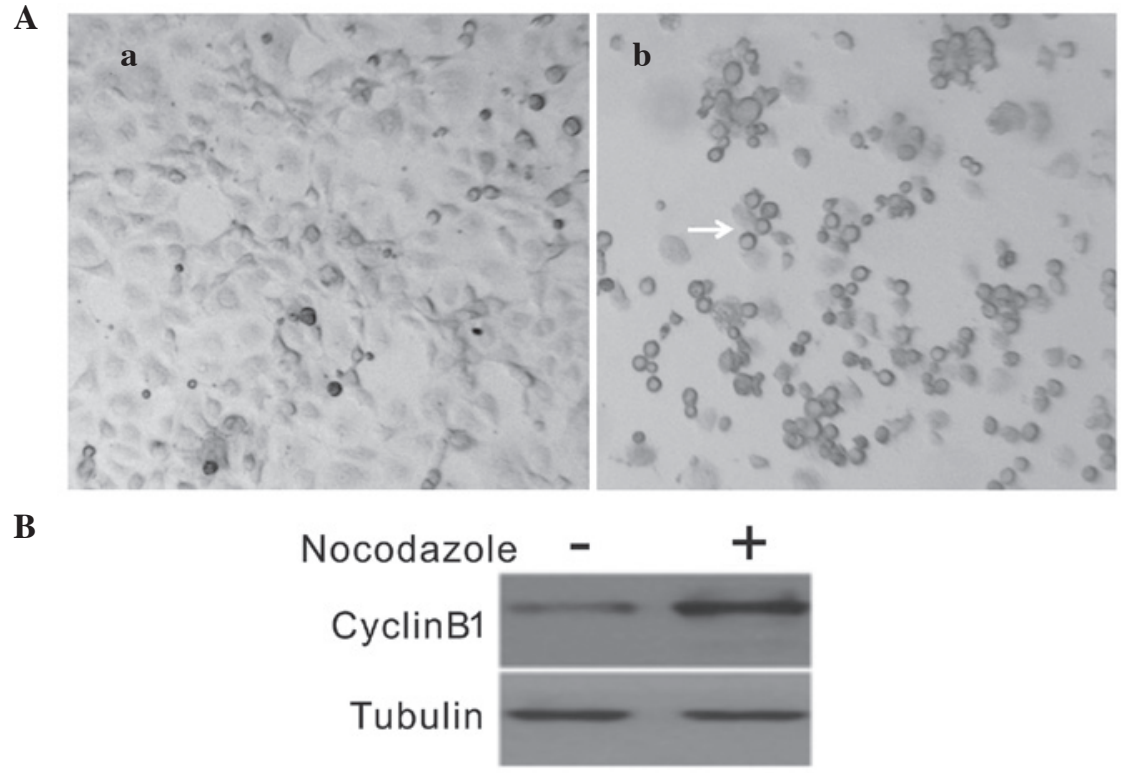

Figure 1. Following treatment for $24 \mathrm{~h}$ with or without nocodazole, COS7 cells were observed under the microscope (magnification, x50). (A-a) Asynchronous (nocodazole-untreated) cells; (A-b) nocodazole-treated cells. Arrows indicate rounded cells. (B) The asynchronous and nocodazole-treated cells were collected and lysed to measure cyclin B1 expression levels. Data are representative of three independent experiments.

(Cwbiotech, Beijing, China), and Akt phosphorylation was detected through the phospho Akt antibody (Santa Cruz Biotechnology, Dallas, TX, USA).

Investigation of the correlation between P85 and EGFR. Following overnight treatment with or without $200 \mathrm{ng} / \mathrm{ml}$ nocodazole, COS7 cells were stimulated with $50 \mathrm{ng} / \mathrm{ml}$ EGF for $15 \mathrm{~min}$, lysed using Mammalian Protein Extraction Kit (Cwbiotech, Beijing, China), and the P85 expression levels were detected through the p85 antibody (Upstate Biotechnology, Inc., Lake Placid, NY, USA). Subsequently, immunoprecipitation experiments were performed to investigate the interaction between P85 and EGFR as described in a previous study (19). Cells were lysed with immunoprecipitation buffer overnight at $4^{\circ} \mathrm{C}$ and the resulting cell lysates were centrifuged at $21,000 \times \mathrm{g}$ for $30 \mathrm{~min}$. The supernatants, containing $1 \mathrm{mg}$ of total protein, were incubated with $1 \mu \mathrm{g}$ of monoclonal mouse anti-EGFR antibody to immunoprecipitate EGFR from COS7 cells. For the control experiments, primary antibodies were replaced with normal mouse or sheep IgG, which caused no EFGR precipitation.

Western blot analysis. Equal amounts of proteins $(20 \mu \mathrm{g})$ were separated by 7.5 or $10 \%$ SDS-PAGE, transferred onto nitrocellulose papers and probed with antibodies against Akt,
phospho-Akt, EGFR, phospho-EGFR, p85, Cyclin B1 and Tubulin. The band intensity of phospho Akt (P-Akt) was quantified using Image J software (National Institutes of Health, USA).

Wound-healing assay. COS7 cells $\left(5.0 \times 10^{6}\right)$ were seeded into six-well plates and grown to confluence. The confluent cell monolayer was scratched using a sterile micropipette tip, and the wounded monolayer was washed with PBS to remove cell debris. The monolayer was incubated with or without $40 \mathrm{ng} / \mathrm{ml}$ nocodazole for $4 \mathrm{~h}$. Following incubation, $0.5 \mu \mathrm{M}$ AG1478, $100 \mathrm{nM}$ wortmannin and $1 \mathrm{ng} / \mathrm{ml}$ EGF were added and the cells were cultured for a further $24 \mathrm{~h}$. Cell migration was evaluated by measuring the width of the wounds.

Statistical analysis. In all cases, the data from three independent experiments were evaluated. The results of the western blot analysis were expressed as the mean \pm standard error of triplicate measurements. All data were analyzed with the software package SPSS 16.0 (SPSS, Inc., Chicago, IL, USA). P<0.05 was considered to indicate a statistically significant difference.

\section{Results}

Nocodazole affects the cell cycle. Nocodazole affects cell morphology and arrests cells in the $\mathrm{G}_{2} / \mathrm{M}$ phase (20). The results 
A
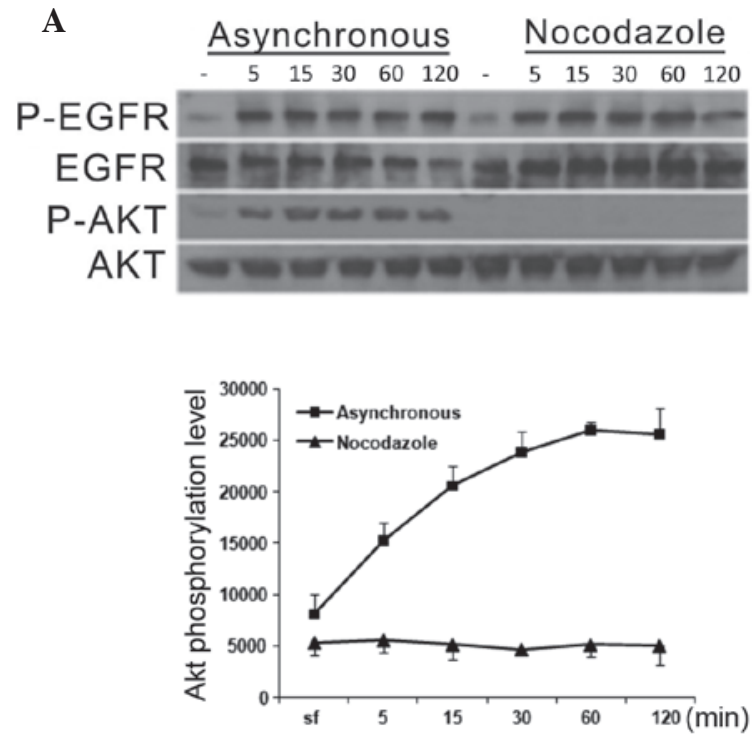

B Asynchronous Nocodazole $\mathrm{EGF} / \mathrm{min}$

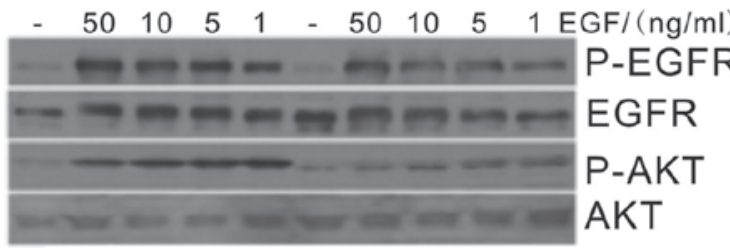

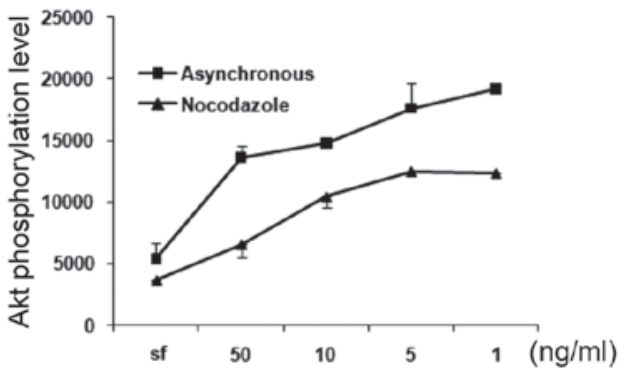

Figure 2. Asynchronous (control) and nocodazole-treated cells were treated (A) without or with EGF (50 ng/ml) for 5, 15, 30, 60 and 120 min and (B) for $15 \mathrm{~min}$ without or with different concentrations of EGF $(1,5,10$ or $50 \mathrm{ng} / \mathrm{ml})$. Active EGFR and Akt (P-EGFR and P-Akt) were analyzed by immunoblotting. Representative results are shown. The band intensity of P-Akt was shown by Image $\mathbf{J}$ software. Data are presented as the mean \pm standard error of three separate experiments.
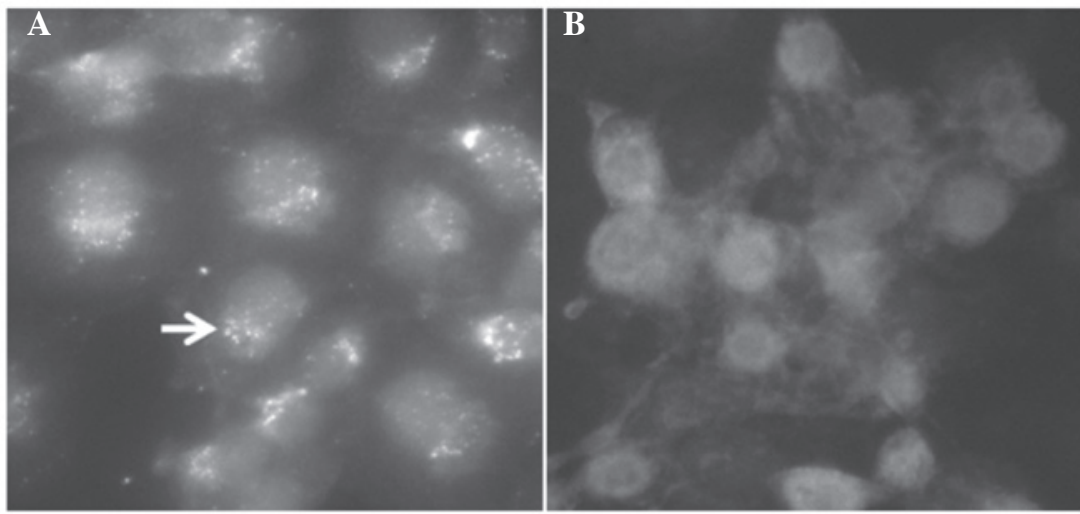

$\mathbf{C}$

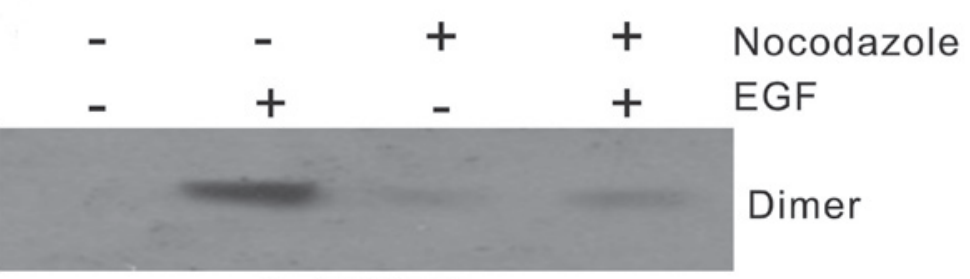

Anti-EGFR

Figure 3. Endocytosis of EGF-EGFR complexes. COS7 cells were treated for $24 \mathrm{~h}$ (A) without or (B) with nocodazole, and subsequently treated with TR-EGF for $30 \mathrm{~min}$. The cells were fixed with methanol and acetone for $10 \mathrm{~min}$ at $-20^{\circ} \mathrm{C}$ and the endosomes were then observed using a fluorescence microscope. Arrows indicate endosomes. (C) 293T cells were transiently transfected with EGFR-YFP. After EGF stimulation for 15 min, 293T cells were cross-linked with bis-(sulfosuccinimidyl suberate). The cell lysates were immunoblotted with an anti-EGFR antibody.

of the current study demonstrated that nocodazole-treated COS7 cells were rounder in appearance and the cell morphology changed markedly compared with that of the untreated cells (Fig. 1A). Following treatment, COS7 cells were collected to examine the expression of cyclin $\mathrm{B} 1$, a protein expressed predominantly during the $\mathrm{G}_{2} / \mathrm{M}$ stage. The results demonstrated that the expression levels of cyclin B1 were markedly increased in nocodazole-treated cells compared with the control cells (Fig. 1B). These results suggest that the cell cycle was affected by nocodazole. To determine the phases of cell cycle in detail, COS7 cells were collected and the cell cycle was analyzed using flow cytometry. In nocodazole-treated cells, the percentages of COS7 cells in the $\mathrm{G}_{0} / \mathrm{G}_{1}, \mathrm{~S}$ and $\mathrm{G}_{2} / \mathrm{M}$ phases were 17.8, 36.7 and $45.5 \%$, respectively. However in nocodazole-untreated cells, the percentages of COS7 cells in $\mathrm{G}_{0} / \mathrm{G}_{1}, \mathrm{~S}$ and $\mathrm{G}_{2} / \mathrm{M}$ phase were $30.6,61.2$ and $8.2 \%$, respec- 
A

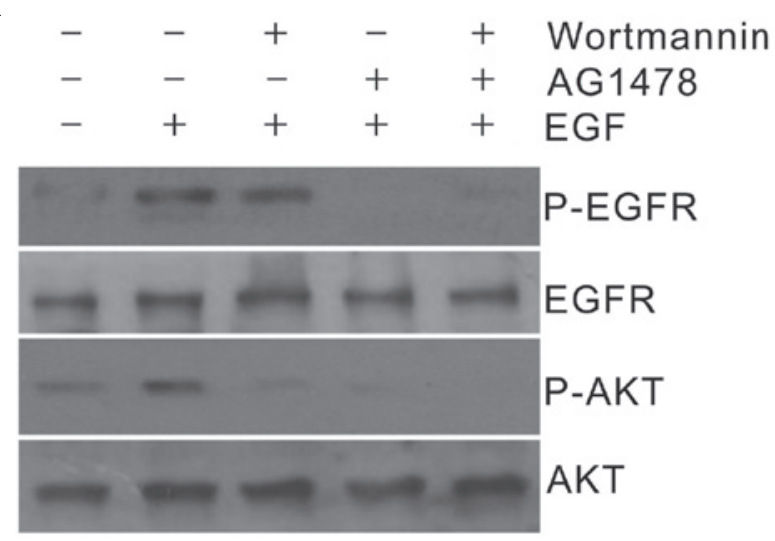

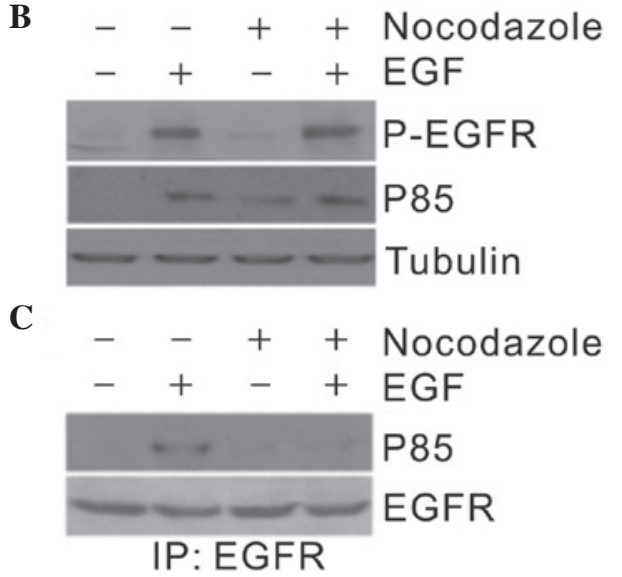

Figure 4. Akt upstream protein activity in nocodazole-treated cells. (A) Asynchronous (control) and nocodazole-treated COS7 cells were treated with $0.5 \mu \mathrm{M}$ AG1478 or $100 \mathrm{nM}$ wortmannin for $30 \mathrm{~min}$, followed by treatment with or without EGF for 15 min. Expression levels of phosphorylated (P)-EGFR and P-Akt were analyzed by immunoblotting. (B) Following overnight treatment with or without $200 \mathrm{ng} / \mathrm{ml}$ nocodazole, COS7 cells were stimulated with $50 \mathrm{ng} / \mathrm{ml}$ EGF for 15 min by followed by lysis of the cells and detection of the P85 expression levels. (C) COS7 cells were treated with or without nocodazole for 24 h, followed by treatment with or without EGF for $15 \mathrm{~min}$. The cells were lysed and immunoprecipitated (IP) with an anti-EGFR antibody and immunoprecipitates were subjected to immunoblotting with anti-EGFR and anti-p85 antibodies.

tively. There were significant discrepancies in the content of the different phases of the cell cycle between nocodazole-treated cells and nocodazole-untreated cells $(\mathrm{P}<0.05$, Table I).

Akt phosphorylation is downregulated in nocodazole-treated cells. Cell cycle progression is associated with innate cell signaling. When the cells are stimulated by EGF, Akt activity is clearly regulated. COS7 cells were stopped at the $\mathrm{G}_{2} / \mathrm{M}$ phase by nocodazole, stimulated by $50 \mathrm{ng} / \mathrm{ml} \mathrm{EGF}$ and the levels of phosphorylation of EGFR and Akt were detected. The results demonstrated that EGFR in nocodazole-untreated and -treated cells was greatly phosphorylated by EGF (Fig. 2A). EGF-mediated Akt phosphorylation in nocodazole-treated cells was almost completely inhibited, whereas EGF-mediated Akt phosphorylation in the asynchronous control cells was gradually increased with the elongation of EGF (Fig. 2A).

Additionally, the effect of EGF concentration on Akt phosphorylation was analyzed. EGF gradients were employed, including 1, 5, 10 and $50 \mathrm{ng} / \mathrm{ml}$, to stimulate COS7 cells. The results demonstrated that Akt phosphorylation level was reduced by $50 \mathrm{ng} / \mathrm{ml}$ EGF stimulation for $15 \mathrm{~min}$ (Fig. 2B). The Akt phosphorylation level gradually increased with a reduction in concentration of EGF with the highest levels at $1 \mathrm{ng} / \mathrm{ml} \mathrm{EGF}$ (Fig. 2B).

Endocytosis of EGFR is blocked in nocodazole-treated cells. Receptor-mediated endocytosis is an endocytotic mechanism through which cells internalize membrane receptors, thereby regulating the uptake of receptor signaling at the plasma membrane. EGFR is specifically internalized after binding to its ligand,EGF (21). Following the overnight treatment of cells with nocodazole and a 30-min treatment with TR-EGF, endosomes were barely detectable in the cytoplasm of COS7 cells (Fig. 3B). However, the endosomes in nocodazole-untreated cells were easily observed in the presence of EGF for $30 \mathrm{~min}$ (Fig. 3A). Since EGFR dimerization is a crucial post-ligand-binding event in the regulation of EGFR internalization, EGFR dimerization was subsequently analyzed through a cross-linking

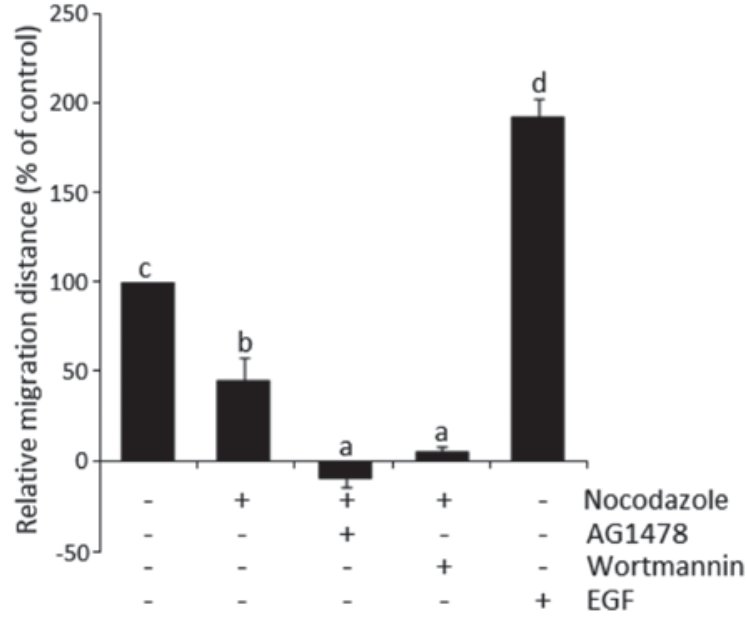

Figure 5. Migration in the presence of nocodazole. A confluent COS7 cell monolayer was scratched with a sterile micropipette tip. The cells were cultured with or without nocodazole $(40 \mathrm{ng} / \mathrm{ml})$ for $4 \mathrm{~h}$ and subsequently treated with EGF $(100 \mathrm{ng} / \mathrm{ml})$, AG1478 $(0.5 \mu \mathrm{M})$ or wortmannin $(100 \mathrm{nM})$. The nocodazole-untreated cells were used as the control group. Wounding data are expressed as a percentage of the migration distance of the nocodazole-untreated cells in $24 \mathrm{~h}$. Different lowercase letters between bars represent $\mathrm{P}<0.05$, while the same letters represent $\mathrm{P}>0.05$.

assay followed by immunblotting. The results showed that the dimerization of EGFR in nocodazole-treated cells was blocked, whereas EGFR in nocodazole-untreated cells was markedly dimerized following EGF stimulation for $15 \mathrm{~min}$ (Fig. 3C).

Akt upstream signaling is reduced in nocodazole-treated cells. To further elucidate the mechanism of Akt phosphorylation inhibition in nocodazole-treated cells, protein inhibitors that are involved in PI3K signal pathway were firstly employed. Treatment of COS7 with wortmannin did not affect EGF-mediated EGFR phosphorylation levels. Nevertheless, EGF-mediated Akt phosphorylation was markedly reduced by wortmannin (Fig. 4A). Additionally, the results demonstrated that EGF-mediated EGFR and 
Akt phosphorylation was blocked by AG1478 (Fig. 4A). Furthermore, it was revealed that the combination of AG1478 and wortmannin gave COS7 cells an increased resistance to EGFR and Akt phosphorylation compared with the untreated control cells (Fig. 4A).

In addition, the results demonstrated that P85 was clearly expressed in nocodazole-treated cells compared with that in the asynchronous controls (Fig. 4B). The interaction between p85 and EGFR in nocodazole-treated cells was subsequently analyzed. P85 is a regulatory subunit of PI3K. When p85 binds to phosphorylated EGFR, the PI3K signaling pathway is activated, which stimulates Akt phosphorylation. The results of the present study demonstrated that p85 readily binds to phosphorylated EGFR in asynchronous cells. However, the binding of p85 to phosphorylated EGFR was reduced in nocodazole-treated cells (Fig. 4C).

Treatment with nocodazole affects cell apoptosis and migration. As Akt phosphorylation was affected in the $\mathrm{G}_{2} / \mathrm{M}$ phase, it is possible that this affects the physiological activities of cells. The results of the current study demonstrated that cell apoptosis was increased in the presence of nocodazole (data not shown). The wound healing assay was used to investigate the migration of cells and the results demonstrated that the relative migration distance in nocodazole-treated cells was significantly less than that of the control $(\mathrm{P}<0.05$, Fig. 5). AG1478 and wortmannin markedly reduced the relative migration distances, particularly in the nocodazole-AG1478 group. Nevertheless, in the presence of EGF, the relative migration distance of nocodazole-untreated cells was significantly increased compared with that of the control cells $(\mathrm{P}<0.05$, Fig. 5).

\section{Discussion}

In spite of efforts to understand the effect of the antimitotic agent nocodazole on cells, a number questions remain unanswered. It has been hypothesized that Akt activity is inhibited by nocodazole, however, the mechanism of action is not well understood. In the present study, EGF was used as a tool to investigate the PI3K/Akt signaling pathway in nocodazole-treated cells.

Previous studies have indicated that nocodazole is primarily involved in the synchronization of the cell division cycle $(20,22,23)$. Cells treated with nocodazole have been observed to arrest with G2- or M-phase DNA content (20). In the current study, a number of cells were observed with a rounded appearance in response to nocodazole treatment and the expression of cyclin B1 was shown to be markedly increased, which revealed that cells entered into the mitosis cycle and arrested at the G2/M phase. Furthermore, the results revealed that the number of cells in $\mathrm{G} 2 / \mathrm{M}$ phase was significantly increased in response to nocodazole compared with those observed in the untreated control cells $(\mathrm{P}<0.05)$. These results show that cell morphology and cell cycle are affected by nocodazole. Therefore, it is proposed that nocodazole may alter the innate cell signaling pathways.

The PI3K/Akt signaling pathway was subsequently investigated in nocodazole-treated cells. A previous study reported that Akt activity was minimal in the cells that had arrested at the G2/M phase due to treatment with nocodazole (11). Hence, nocodazole was employed to treat cells in order to investigate Akt activity. The results demonstrated that EGF-mediated Akt phosphorylation was markedly reduced in nocodazole-treated cells. Akt activity is required for the $\mathrm{G}_{2} / \mathrm{M}$ phase transition $(11,24)$, activated Akt shortens the $\mathrm{G}_{2}$ arrest induced by DNA damage and promotes early cell entry into mitosis (12). The results of the current study revealed that Akt activity was blocked when cells arrested in $\mathrm{G}_{2} / \mathrm{M}$ phase in response to nocodazole. At the same time, it was determined that EGF-mediated Akt phosphorylation in the asynchronous cells was increased with prolonged EGF stimulation, demonstrating that nocodazole resists Akt activity. Additionally, the Akt phosphorylation level was associated with the concentration of EGF treatment, which provided the evidence for determining the precise dosage of EGF stimulation that was required. Whether Akt is significantly activated by low doses of EGF requires further analysis. To summarize, Akt is an important downstream effector of the PI3K pathway, the phosphorylation of which is affected by treatment with nocodazole.

Previous studies have shown that internalized EGF-EGFR complexes are enzymatically active, and maintain their abilities to associate with various signaling molecules and generate cell signals from the endosomes (25). Receptor-mediated EGFR endocytosis selectively regulates the EGFR signaling pathway by changing EGFR localization and rendering signaling molecules available. In the present study, internalized EGF-EGFR complexes were rarely observed in nocodazole-treated cells. It was shown that the EGF-EGFR complexes were blocked from the cytoplasm, thereby attenuating innate cell signals. To further explain this event, the dimerization of EGFR was investigated using a cross-linking assay. The levels of EGFR dimerization in nocodazole-treated cells were markedly reduced compared with those in the control group (Fig. 3B). Therefore, it was concluded that internalized EGF-EGFR complexes are reduced following the inhibition of EGFR in the presence of nocodazole. In addition, this may affect cell signaling from the endosome, for example, endocytosis blocking experiments have shown that endocytosis may be necessary for ERK activation, as it allows MEK1 access to ERK within an intracellular compartment $(26,27)$.

To explore the causes of the reduction of Akt activity, Akt upstream proteins were investigated. It has previously been determined that the PI3K/Akt signaling pathway is initiated by activated EGFR (28). The results of the current study showed that AG1478 effects EGFR activity, hence significantly attenuating Akt phosphorylation. It appears that EGFR phosphorylation is an important process in the activation of the PI3K/Akt signaling pathway. However, the results revealed that Akt phosphorylation was greatly inhibited by nocodazole, although EGFR phosphorylation levels in nocodazole-treated cells were high. Thus, it may be the case that signaling pathways downstream of EGFR were overridden. PI3K activity was investigated through the use of wortmannin. Wortmannin is a specific inhibitor of PI3K, and it significantly blocks PI3K activation by binding to the P110 catalytic subunit of PI3K. When cells were treated with EGF, the phosphorylation levels of EGFR and Akt were increased. It may be that activated EGFR stimulates PI3K activity and causes Akt phosphorylation. However, in wortmannin-treated 
cells EGF-mediated Akt phosphorylation was overridden in spite of high levels of activated EGFR, indicating that the PI3K/Akt signaling pathway was inhibited due to a reduction in PI3K activity. A previous study demonstrated that PI3K activation was associated with EGFR expression and that the regulatory subunit of PI3K, P85, could activate PI3K by binding activated EGFR (29). The current study showed that when COS7 cells were treated with nocodazole, P85 binding to EGFR was less efficient, and Akt phosphorylation was inhibited due to nocodazole-induced PI3K inactivation. A possible cause of this is that the EGF-EGFR complexes were blocked from the cytoplasm by nocodazole, which attenuated the ability of P85 to bind to EGFR. From the results of this study, it may be hypothesized that nocodazole may restrict the PI3K/Akt signaling pathway by affecting the interaction between p85 and activated EGFR. Certainly, the mechanism of action requires further study.

The current study revealed that nocodazole-treated cells show features of apoptotic-like cell death, which may be involved in the PI3K/Akt signaling pathway. Akt is an important mediator of the anti-apoptotic factors in the PI3K pathway. Activated Akt promotes cell survival by phosphorylating and inactivating the forkhead family of transcription factors, by themselves inducing the expression of proapoptotic factors such as the Fas ligand (30). Additionally, Akt phosphorylates the proapoptotic factor BAD, a BCL-2 family member, and suppresses BAD-mediated cellular apoptosis (31). Since cell apoptosis develops, certain apoptotic factors must be activated, which may interfere with cell physiology such as cell migration. Furthermore, previous reports have shown that Akt regulates cell motility $(4,32)$. Hence, in the present study, a wound healing assay was implemented employing inhibitors of cell motility. The results showed that cell migration was greatly affected in the nocodazole, AG1478 and wortmannin treatment groups compared with that of the cells in the control group, demonstrating that Akt activity is important to cell motility.

In conclusion, this study has provided direct molecular evidence that the PI3K/Akt signaling pathway is inhibited by nocodazole via interruption of the binding of $\mathrm{p} 85$ to activated EGFR. Additionally, this study demonstrated the effect of treatment with nocodazole and inhibitors associated to PI3K/Akt pathway on cell apoptosis and migration. Given the high frequency of PI3K inactivation by nocodazole in cancer cells, this study strongly suggests that nocodazole will help to suppress cancer.

\section{Acknowledgements}

This paper was supported by the grants 'Study of EGF-mediated signal pathways in cell mitosis' from the Northwest A\&F University Foundation of China (QN2011010) and 'Effects of PI3K/Akt signal pathway on dairy goat mammary epithelial cells' from the Postdoctoral science foundation of China (2012M512038).

\section{References}

1. Scheid M, Marignani P and Woodgett J: Multiple phosphoinositide 3-kinase-dependent steps in activation of protein kinase B. Mol Cell Biol 22: 6247-6260, 2002.
2. Troussard AA, Mawji NM, Ong C, Mui A, St -Arnaud R and Dedhar S: Conditional knock-out of integrin-linked kinase demonstrates an essential role in protein kinase B/Akt activation. J Biol Chem 278: 22374-22378, 2003.

3. Sarbassov DD, Guertin DA, Ali SM and Sabatini DM: Phosphorylation and regulation of Akt/PKB by the rictor-mTOR complex. Science 307: 1098-1101, 2005.

4. Yoeli-Lerner M and Toker A: Akt/PKB signaling in cancer: a function in cell motility and invasion. Cell Cycle 5: 603-605, 2006.

5. Cantley L: The phosphoinositide 3-kinase pathway. Science 296: 1655-1657, 2002.

6. Partovian C and Simons M: Regulation of protein kinase B/Akt activity and Ser 473 phosphorylation by protein kinase Calpha in endothelial cells. Cell Signal 16: 951-957, 2004.

7. Datta S, Brunet A and Greenberg M: Cellular survival: a play in three Akts. Genes Dev 13: 2905-2927, 1999.

8. Madrid L, Mayo M, Reuther J and Baldwin A, Jr: Akt stimulates the transactivation potential of the RelA/p65 Subunit of NF-kappa B through utilization of the Ikappa B kinase and activation of the mitogen-activated protein kinase p38. J Biol Chem 276: 18934-18940, 2001

9. Franke T, Hornik C, Segev L, Shostak G and Sugimoto C: PI3K/Akt and apoptosis: size matters. Oncogene 8: 8983-8998, 2003.

10. Liang $\mathrm{J}$ and Slingerland JM: Multiple roles of the PI3K/PKB (Akt) pathway in cell cycle progression. Cell Cycle 2: 339-345, 2003.

11. Lee S, Park J, Park E, Chung C, Kang S and Bang O: Akt-induced promotion of cell-cycle progression at $\mathrm{G} 2 / \mathrm{M}$ phase involves upregulation of NF-Y binding activity in PC12 cells. J Cell Physiol 205: 270-277, 2005.

12. Shtivelman E, Sussman J and Stokoe D: A role for PI 3-kinase and PKB activity in the $\mathrm{G} 2 / \mathrm{M}$ phase of the cell cycle. Curr Biol 12: 919-924, 2002.

13. Li Q and Zhu G: Targeting serine/threonine protein kinase B/Akt and cell-cycle checkpoint kinases for treating cancer. Curr Top Med Chem 2: 939-971, 2002.

14. Mahimainathan L, Ghosh-Choudhury N, Venkatesan BA, Danda RS and Choudhury GG: EGF stimulates mesangial cell mitogenesis via PI3-kinase-mediated MAPK-dependent and AKT kinase-independent manner: involvement of c-fos and p27Kip1. Am J Physiol Renal Physiol 289: F72-F82, 2005.

15. Woscholski R, Kodaki T, McKinnon M, Waterfield MD and Parker PJ: A comparison of demethoxyviridin and wortmannin as inhibitors of phosphatidylinositol 3-kinase. FEBS Lett 342: 109-114, 1994.

16. Takesono A, Heasman SJ, Wojciak-Stothard B, Garg R and Ridley AJ. Microtubules regulate migratory polarity through Rho/ROCK signaling in T cells. PLoS One 5: e8774, 2010.

17. Kim SH, Juhnn YS and Song YS: Akt involvement in paclitaxel chemoresistance of human ovarian cancer cells. Ann N Y Acad Sci 1095: 82-89, 2007.

18. Hemstrom TH, Sandstrom M and Zhivotovsky B: Inhibitors of the PI3-kinase/Akt pathway induce mitotic catastrophe in non-small cell lung cancer cells. Int J Cancer 119: 1028-1038, 2006.

19. Wang Z,Zhang L, Yeung TK and Chen X: Endocytosis deficiency of epidermal growth factor (EGF) receptor-ErbB2 heterodimers in response to EGF stimulation. Mol Biol Cell 10: 1621-1636, 1999.

20. Tran T, Kolupaeva V and Basilico C: FGF inhibits the activity of the cyclin B1/CDK1 kinase to induce a transient G(2)arrest in RCS chondrocytes. Cell Cycle 9: 4379-4386, 2010.

21. Ajioka RS and Kaplan J: Intracellular pools of transferrin receptors result from constitutive internalization of unoccupied receptors. Proc Natl Acad Sci U S A 83: 6445-6449, 1986.

22. Dangi S and Shapiro P: Cdc2-mediated inhibition of epidermal growth factor activation of the extracellular signal-regulated kinase pathway during mitosis. J Biol Chem 280: 24524-24531, 2005.

23. Shi $H$ and Shen L: The alteration of extracellular signal-regulated kinase (ERK) activity of COS7 cells from interphase to mitosis. Cell Mol Biol (Noisy-le-grand) 56 Suppl: OL1427-1433, 2010

24. Dangi S, Cha H and Shapiro P: Requirement for phosphatidylinositol-3 kinase activity during progression through S-phase and entry into mitosis. Cell Signal 15: 667-675, 2003. 
25. Wang Y, Pennock S, Chen X and Wang Z: Endosomal signaling of epidermal growth factor receptor stimulates signal transduction pathways leading to cell survival. Mol Cell Biol 22: 7279-7290, 2002.

26. Haugh JM, Huang AC, Wiley HS, Wells A and Lauffenburger DA: Internalized epidermal growth factor receptors participate in the activation of p21(ras) in fibroblasts. J Biol Chem 274: 34350-34360, 1999.

27. Tong X, Hussain N, Adams A, O'Bryan J and McPherson P: Intersectin can regulate the Ras/MAP kinase pathway independent of its role in endocytosis. J Biol Chem 275: 29894-29899, 2000.

28. Blair K, Kiang A, Wang-Rodriguez J, Yu M, Doherty J and Ongkeko W: EGF and bFGF promote invasion that is modulated by PI3Akt kinase and Erk in vestibular schwannoma. Otol Neurotol 32: 308-314, 2011
29. Dougall WC, Qian X, Miller MJ and Greene MI: Association of signaling proteins with a nonmitogenic heterodimeric complex composed of epidermal growth factor receptor and kinase-inactive p185c-neu. DNA Cell Biol 15: 31-40, 1996.

30. Brunet A, Bonni A, Zigmond MJ, et al: Akt promotes cell survival by phosphorylating and inhibiting a Forkhead transcription factor. Cell 96: 857-868, 1999.

31. Datta SR, Dudek H, Tao X, et al: Akt phosphorylation of BAD couples survival signals to the cell-intrinsic death machinery. Cell 91: 231-241, 1997.

32. Enomoto A, Murakami H, Asai N, et al: Akt/PKB regulates actin organization and cell motility via Girdin/APE. Dev Cell 9: 389-402, 2005. 